Rev. Adm. Saúde (On-line), São Paulo, v. 19, n. 76: e179, jul. - set. 2019, Epub 09 set. 2019

http://dx.doi.org/10.23973/ras.76.179

RELATO DE CASO

\title{
Reestruturação de núcleo de segurança do paciente com aplicação da metodologia lean seis sigma
}

Patient safety nucleus restructuring with lean six sigma methodology application

\author{
Andre Barros Ferreira ${ }^{1}$, Ricardo Rodrigues de Castro Teixeira ${ }^{2}$
}

1. Biomédico, especialista em gestão da qualidade. Gerente da Oculare - Hospital de Oftalmologia, Belo Horizonte MG

2. Graduado em gestão da qualidade. Coordenador da Qualidade da Oculare - Hospital de Oftalmologia, Belo Horizonte MG

\section{RESUMO}

Em uma época em que o tema segurança do paciente vem sendo desenvolvido sistematicamente por órgãos de fiscalização e qualidade, os hospitais e clínicas devem cada dia mais ampliar a cultura de segurança do paciente. Para fazer isso, é necessário adotar medidas estratégicas e ferramentas de gestão LEAN, inspiradas em práticas de gestão e resultados do sistema Toyota. Este artigo relata a implantação de ferramentas LEAN no Núcleo de Segurança do Paciente (NSP) em um hospital de olhos de médio porte. Os objetivos da implantação eram: elevar a cultura de segurança do paciente na instituição e aumentar as notificações de possíveis eventos (near miss) a fim de garantir ação prévia e evitar danos; em paralelo, diminuir subnotificações e o tempo de ação dos membros do NSP frente às notificações, facilitando o acesso das notificação aos colaboradores e, desta forma, permitir a prevenção de eventos adversos com dano. A implantação foi desenvolvida em cinco fases, que vão da concepção do projeto até a análise dos resultados. Foram evidenciadas melhorias na compreensão da finalidade do NSP, ampliando de $82 \%$ para $100 \%$ o conhecimento sobre o Núcleo por parte dos colaboradores. Os resultados mostraram a importância na utilização das ferramentas Jidoka e Kaizen, fomentando a criação do website do NSP que permitiu ampliar os conhecimentos dos colaboradores e diminuir de $77 \%$ para $3 \%$ a dificuldade em 
notificar. O sistema de envio de e-mail imediato aos membros melhorou o tempo de ação do NSP, reduzindo a média de 27 dias para apenas 1 dia. Observamos que, mesmo em época de crise, com pequeno custo, houve um aumento da cultura de segurança do paciente no hospital em 357,14\% em comparação com o ano anterior, com aumento de notificações de near miss (eventos que podem ser controlados para não causarem dano). Hoje em dia, a aplicação de ferramentas e conceitos LEAN nas estratégias gerenciais é garantia fundamental da sustentabilidade, cumprimento de normas e leis, sem que haja impacto financeiro que impeça a garantia da segurança do paciente.

Palavras-chave: Segurança do paciente. Administração hospitalar. Gestão de riscos. Eficiência organizacional. Indicadores de qualidade em assistência à saúde.

\section{ABSTRACT}

At a time when the subject of patient safety has been systematically developed by oversight and quality agencies, hospitals and clinics must increasingly broaden the patient safety culture. To do this, strategic measures and LEAN management tools, based on Toyota system management practices and results, must be adopted. This article reports the implementation of LEAN tools at the Patient Safety Center (NSP) in a medium-sized eye hospital. The objectives of the implantation were: to elevate the patient safety culture in the institution and increase the notifications of possible events (near miss) in order to guarantee previous action and avoid damages; in parallel, reduce underreporting and action time of NSP members in the face of notifications, facilitating the access of notifications to employees and, thus, allowing the prevention of adverse events with damage. The deployment was developed in five phases, ranging from project design to results analysis. Improvements in the understanding of the purpose of the NSP were evidenced, increasing from $82 \%$ to $100 \%$ the knowledge about the Center by the employees. The results showed the importance of using the Jidoka and Kaizen tools, promoting the creation of the NSP website that allowed the employees to increase their knowledge and reduce from $77 \%$ the difficulty to notify. Members' immediate email system has improved NSP action time, reducing the average from 27 days to just 1 day. Even at a time of crisis, at a low cost, there was an increase in the patient safety culture in the hospital by $357.14 \%$ compared to the previous year, with an increase in near miss reports (events that can be controlled for do no harm). Nowadays, the application of LEAN tools and concepts in management strategies is a fundamental guarantee of sustainability, compliance with rules and laws, without having a financial impact that does not guarantee patient safety.

Keywords: Patient Safety. Hospital administration. Risk management. Efficiency, organizational. Quality indicators, Health Care. 


\section{INTRODUÇÃO}

Cada vez mais, hospitais e clínicas têm se preocupado com a segurança do paciente. No Brasil, estudo realizado pela Faculdade de Medicina da Universidade Federal de Minas Gerais (UFMG), aponta que a cada três minutos, mais de dois brasileiros morrem em um hospital público ou privado como consequência de um evento adverso ${ }^{1}$. Em paralelo, ao redor do mundo, a gestão de riscos e a melhoria nos cuidados e na qualidade, passam a ter esforços conjuntos dentro das organizações de saúde, perseguindo a segurança do paciente ${ }^{2,3,4,5,6}$. Nos Estados Unidos, em 2016, uma em cada duas cirurgias apresentava algum erro relacionado à medicação, e estima-se que em cada dez pacientes hospitalizados um desenvolve algum tipo de evento adverso $(E A)^{7,8,9,10}$. Esses erros apresentam grandes riscos aos pacientes, que podem variar entre "possibilidade de erro" (near miss) a "óbito". Além dos fatores de saúde, os EA afetam a imagem da instituição de saúde e aumentam consideravelmente os custos da assistência. O valor médio gasto nos Estados Unidos, em 2015, pelo atendimento aos pacientes com EA foi $200,5 \%$ superior ao valor pago aos pacientes sem $\mathrm{EA}^{11}$.

$\mathrm{Na}$ última década, o tema segurança do paciente vem sendo desenvolvido sistematicamente pela Agência Nacional de Vigilância Sanitária (Anvisa), com a missão de intervir nos riscos advindos do uso de produtos e serviços de saúde bem como o uso das tecnologias disponíveis para o cuidado ${ }^{12,13}$. O Programa Nacional de Segurança do Paciente (PNSP), instituído no Brasil pela Portaria GM n'. 529, de 01 de abril de 2013, juntamente com a publicação da Política Nacional de Atenção Hospitalar (PNHOSP), estabelecem que as instituições de saúde devem garantir a segurança, educação dos colaboradores frente ao tema, e cuidados com a saúde, criando e difundindo a nível institucional o Núcleo de Segurança do Paciente (NSP) ${ }^{14,15}$. Desde 2004, a ANS vem intensificando suas atividades no campo de serviços de saúde em parceria com o Ministério da Saúde (MS), a Organização Pan-Americana da Saúde (Opas) e o Sistema Nacional de Vigilância Sanitária (SNVS) ${ }^{16,17,18 .}$

Evento adverso (EA) trata-se de um evento que poderia ter resultado, ou resultou, em dano desnecessário ao paciente ${ }^{19}$. São classificados em near miss, eventos que não atingiram o paciente, porém oferecem o risco, se não remediados, de causarem danos. Dano leve, evento que é percebido conscientemente pelo paciente (psicológico), é assintomático, não necessitando de tratamento. Dano moderado, onde há consequência no paciente podendo ou não causar danos permanentes ou a longo prazo, ou perda de funções; é sintomático, requerendo intervenção. Dano grave, atingiu o paciente, é sintomático, requer intervenção para salvar a vida. Óbito, morte causada ou antecipada pelo incidente $20,21,22$.

No Brasil, um estudo retrospectivo evidenciou que $66,7 \%$ dos eventos adversos poderiam ser prevenidos caso fossem notificados como near miss antes de se tornarem dano, através de abordagens de segurança e consciência 
institucional ${ }^{23}$. Sabe-se que no Brasil, $80 \%$ dos EA acontecem devido a subnotificação de near miss, transformando o que antes era possibilidade de prevenção em dano ${ }^{24,25}$. A maior dificuldade de uma instituição de saúde é criar em seus colaboradores a cultura com o objetivo de garantir a segurança do paciente, percebendo e notificado near miss, que possam comprometer a segurança gerando dano ao paciente $26,27,28$.

Para acompanhar as mudanças globais que envolvem a segurança de pacientes em hospitais e clínicas, o Núcleo de Segurança do Paciente (NSP) tem como finalidade estabelecer políticas e diretrizes de trabalho a fim de promover uma cultura hospitalar voltada para a segurança dos pacientes, através do planejamento, desenvolvimento, controle e avaliação de programas e tecnologias, que visem garantir a qualidade dos processos na Instituição ${ }^{29}$.

O termo LEAN foi criado na década de 80 a partir de um projeto de pesquisa do Massachusetts Institute of Technology - MIT, sobre a indústria automobilística ${ }^{30,31}$. Trata-se de uma filosofia criada e difundida no sistema de produção Toyota que visa eliminar desperdícios, essa filosofia é comumente chamada de manufatura enxuta ${ }^{32}$. Uma das ferramentas LEAN, chamada Jidoka, permite o uso de equipamentos e sistemas que possam criar uma automação e sinalizar quando há uma não conformidade. O visual factory, mais conhecido pelo sistema da qualidade como "gestão visual", é uma ferramenta importante na divulgação e conscientização de tarefas, normas, leis e processos de forma visual e prática. Outra ferramenta LEAN, chamada Kaizen, permite que talentos das empresas criem mecanismos mecânicos e técnicos para eliminar perdas ${ }^{33,34}$. Os benefícios das três ferramentas podem ser utilizados em estratégias empresariais que podem reduzir erros provenientes de processos, contribuindo para a melhoria da gestão ${ }^{35,36}$.

Objetivo do trabalho. Disseminar e educar, aumentar a cultura de segurança do paciente e as notificações de possíveis eventos (near miss) a fim de garantir ação prévia e evitar danos, diminuir subnotificações e o tempo de ação dos membros do Núcleo de Segurança do Paciente frente às notificações, permitindo assim a prevenção de eventos adversos com dano, através das ferramentas LEAN.

\section{METODOLOGIA}

A metodologia adotada baseia-se nas ferramentas da qualidade, associadas a ferramentas LEAN de manufatura enxuta (Jidoka, Visual Factory e Kaizen). A implantação foi dividida em cinco fases baseadas no ciclo PDCA: projeto,coleta de dados (cenário atual), análise, implantação, resultado. A coleta de dados (análise do cenário atual), foi realizada através da análise de indicadores de EA do Núcleo de Segurança do Paciente em relação a notificações de near miss. Também foi realizada pesquisa com $40,20 \%$ dos colaboradores sobre o Núcleo de Segurança e sistema de notificação, através de um Tablet, utilizando planilha do MS-Excel como banco de dados. Os dados da pesquisa foram analisados no MS- Excel com a estratificação dos problemas relatados pelos colaboradores, bem como números de notificações. Na fase analítica foram 
empregadas as ferramentas de qualidade: $5 \mathrm{~W} 2 \mathrm{H}, 5$ porquês, diagrama de Ishikawa (causa e efeito) e A3. Os resultados da fase analítica, com as oportunidades de melhorias foram discutidas e implantadas de acordo com a matriz de esforço e impacto, através das ferramentas LEAN, acompanhados mensalmente juntos aos resultados, lançados em gráfico de tendência comparativo com os dados iniciais. Na fase de implantação, foram utilizadas ferramentas LEAN (Visual Factory, Jidoka, Kaizen, Gemba Walk, 5s), estas foram testadas e implantadas em todo o hospital. Os colaboradores receberam treinamentos em grupos para cada ferramenta. Na fase de resultados, os indicadores do NSP foram analisados e comparados, estratificados em préLEAN e pós-LEAN. A pesquisa realizada na fase de coleta de dados com $40,20 \%$ dos colaboradores, foi refeita com os mesmos colaboradores. Os resultados lançados em planilhas do MS-Excel e MiniTab18.

\section{RESULTADOS}

Com a implementação da ferramenta Visual Factory, os colaboradores ficaram mais informados sobre o Núcleo de Segurança do Paciente (NSP), diminuindo o índice de $18 \%$ dos colaboradores que não sabiam opinar sobre o assunto para $0 \%$ (melhoria de $82 \%$ para 100\%). A utilização das ferramentas Jidoka e Kaizen, com a criação de site do NSP, permitiu ampliar os conhecimentos dos colaboradores além de facilitar o processo de notificação diminuindo o relato de dificuldades em notificar de $77 \%$ para $3 \%$ dos colaboradores (Figura 1).

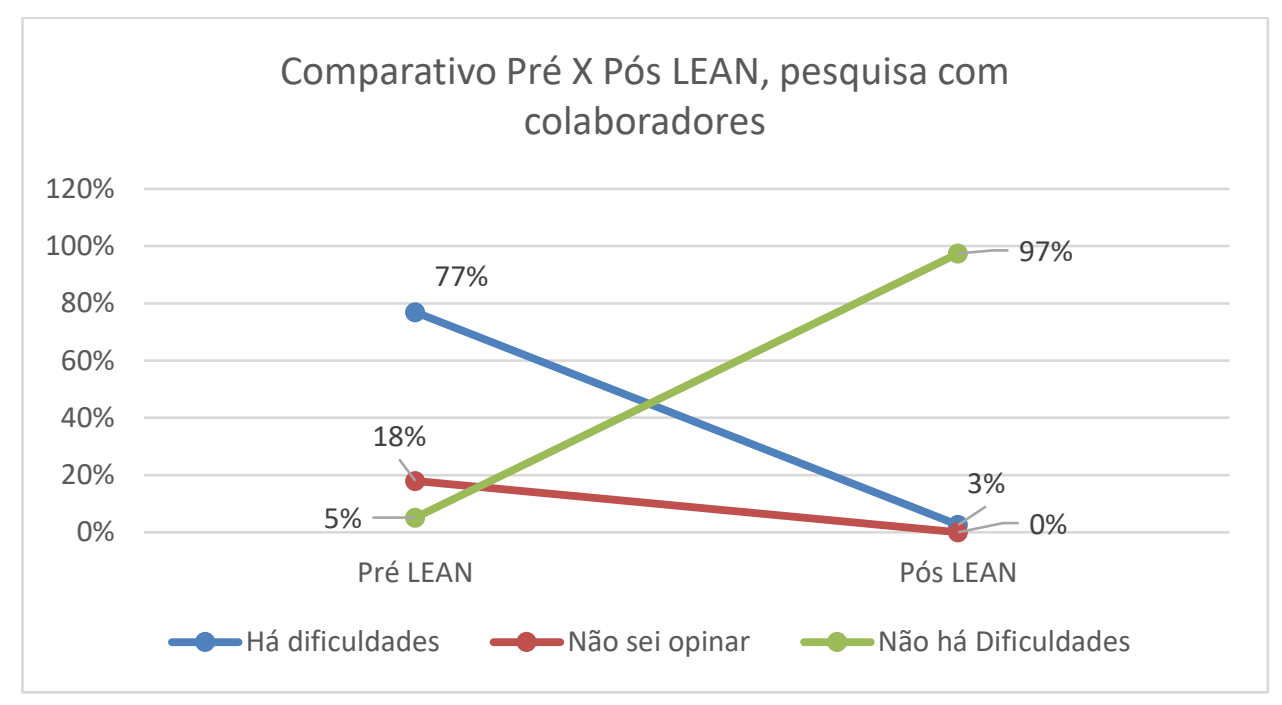

Figura 1. Comparativo da pesquisa com colaboradores, pré e pós LEAN.

O sistema de envio de e-mail imediato aos membros do NSP, assim que fosse registrada uma notificação no site, melhorou o tempo de ação do NSP de uma média de 27 dias para 1 dia (Figura 2). 


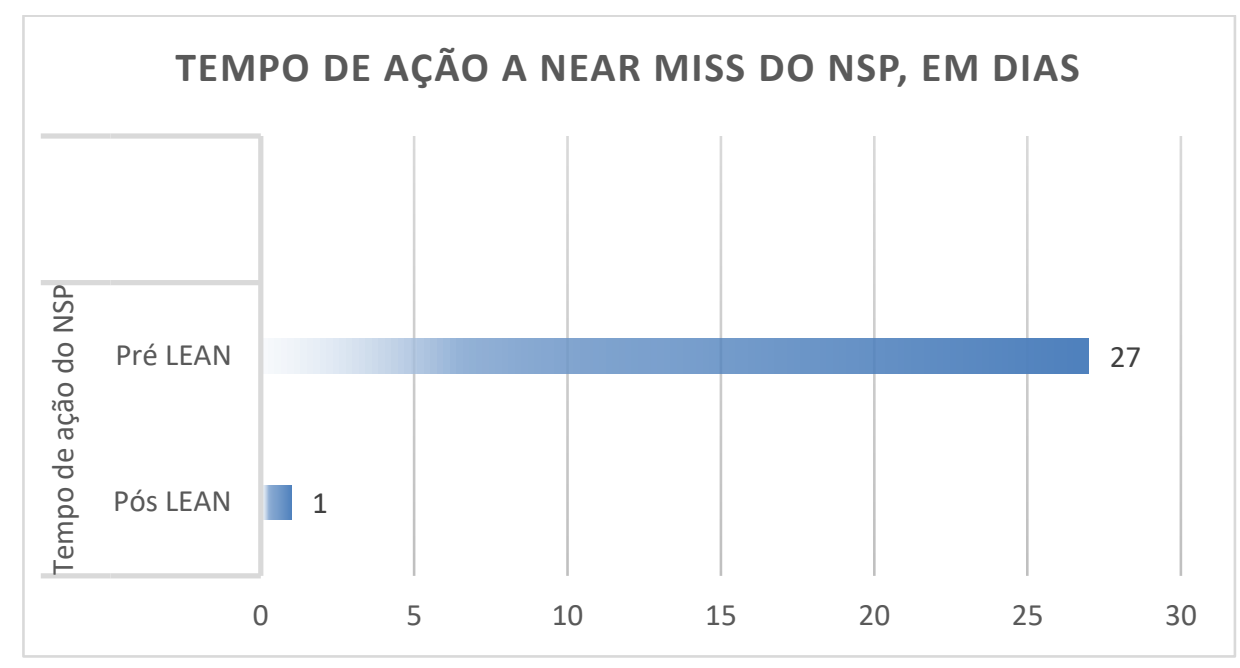

Figura 2. Tempo de Ação a near miss do NSP, em dias.

Com a implantação das ferramentas e medidas, houve um aumento da cultura de segurança do paciente em $357,14 \%$ com aumento das notificações de near miss (eventos que podem ser controlados para não causarem dano), antes subnotificadas, garantindo assim, maior cultura de segurança (Figura 3).

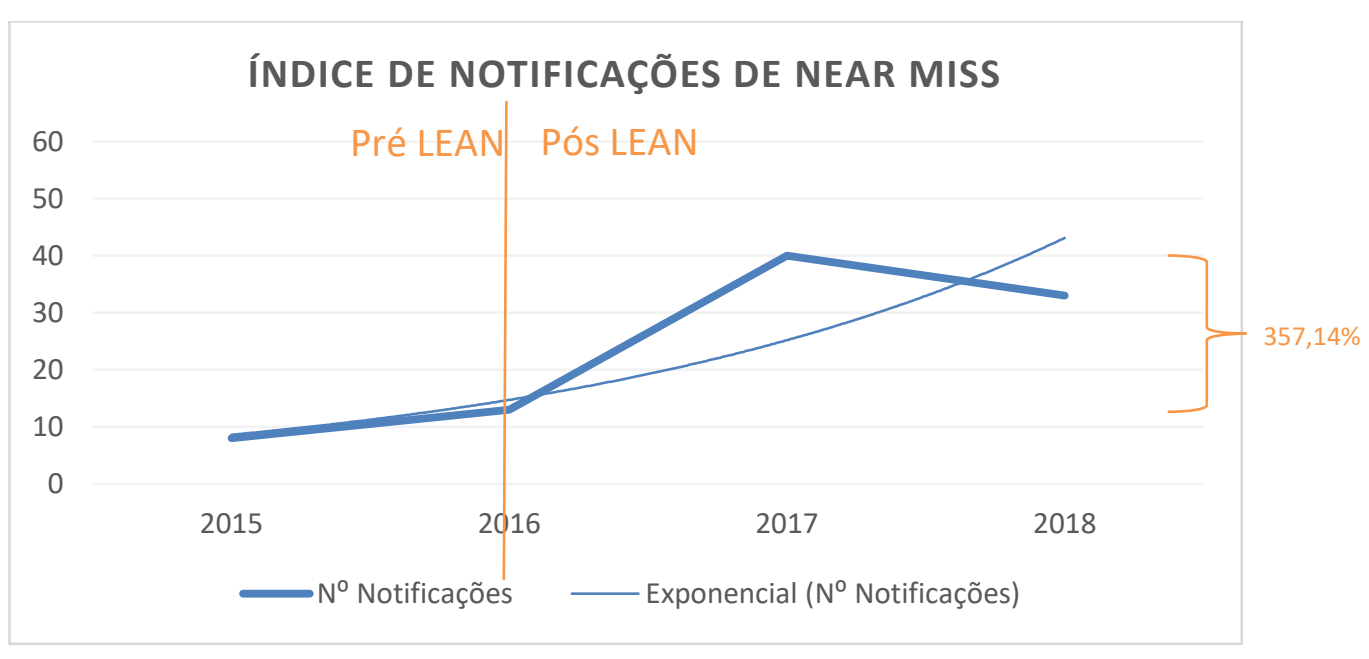

Figura 3. Índice de Notificações near miss.

As ações e ferramentas permitiram sanar as dificuldades dos colaboradores, frente as notificações, normas e identificação de riscos, através de site e gerenciamento visual a um custo final de duzentos reais. Estas ações garantiram a melhoria da cultura de segurança do paciente no Hospital, tornando-o mais seguro e atendendo as normas legais. 


\section{DISCUSSÃO}

A implantação de ferramentas LEAN trata-se não apenas de uma gestão de ferramentas da qualidade, mas sim de mudança na filosofia e cultura requerendo muito envolvimento dos colaboradores e diretoria. O processo iniciou-se com coleta de informações do cenário atual, onde foram apuradas informações relevantes ao alvo do projeto. Nesta etapa pudemos notar que o número de notificações de near miss anual era muito baixo, em 2015 um total de oito notificações e em 2016 um total de treze; índices muito baixos para um atendimento hospitalar 24 horas (Figura 4).

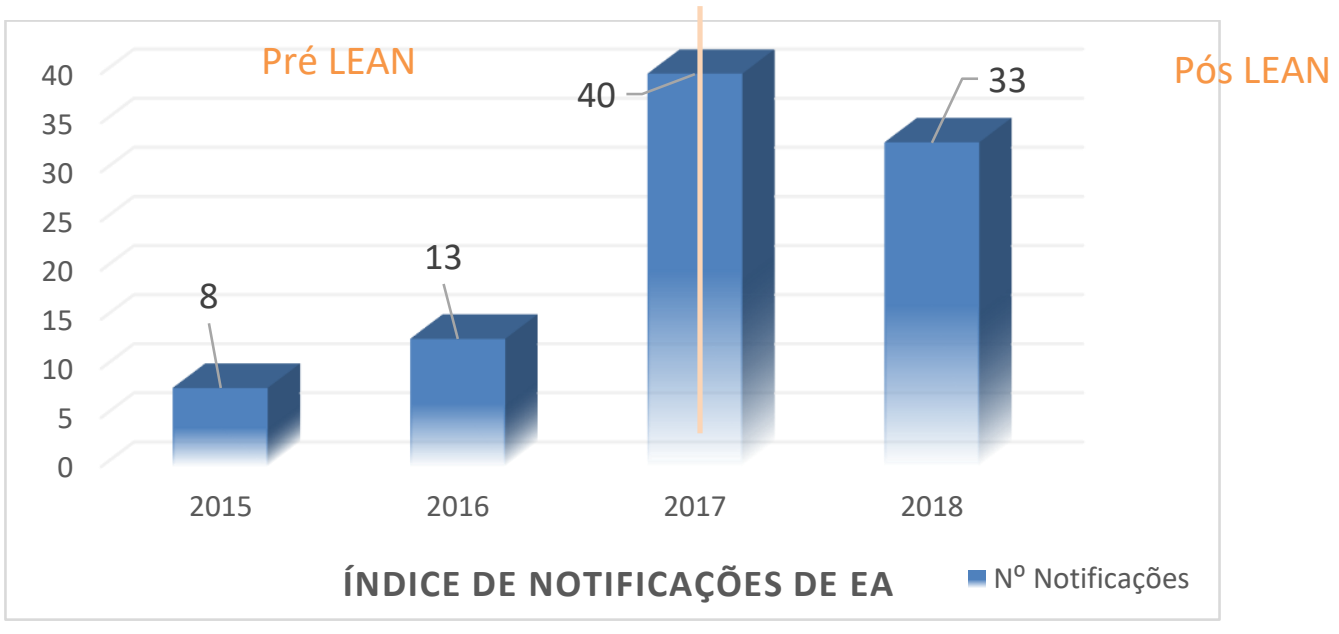

Figura 4. Índice de notificações de near miss.

Em maio de 2017, para analisar o cenário atual, foi realizada pesquisa com $40,20 \%$ dos colaboradores. Em um total de trinta e nove colaboradores, trinta (77\%), informaram que tinham dificuldades em notificar o NSP, sete $(18 \%)$, informaram que não sabiam opinar, dois (5\%) informaram não terem dificuldades. Os colaboradores que relataram dificuldades de notificação e que não sabiam opinar, um total junto de trinta e sete colaboradores (95\%), tiveram suas respostas estratificadas. Dos trinta e sete colaboradores, dois (5\%) não sabiam o que era NSP, cinco (14\%) não sabiam quando deveriam notificar, sete $(19 \%)$ não sabiam notificar, vinte e três (62\%) não achavam prático notificar (Figura 5). 


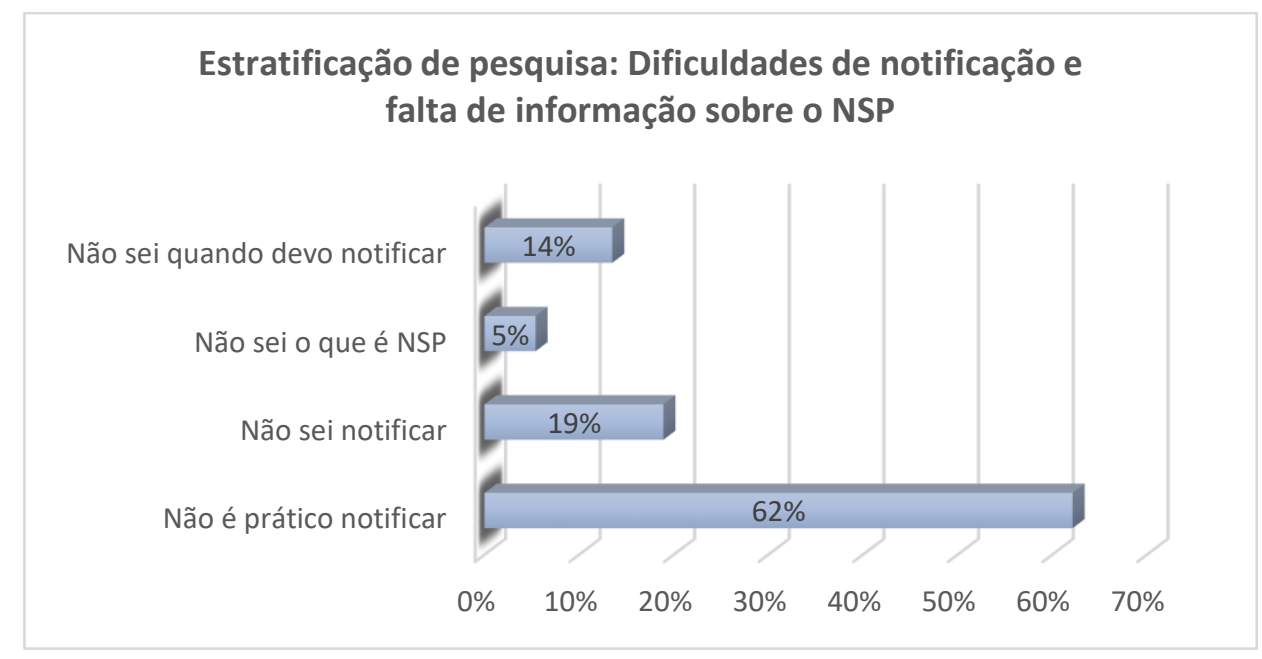

Figura 5. Estratificação de dificuldades de notificação e falta de informação sobre o NSP

Em vista do resultado, utilizando ferramentas de qualidade, chegamos à conclusão de que havia falhas na divulgação do NSP, na praticidade de notificação, no treinamento da equipe e no entendimento dos eventos adversos (o que notificar?).

A primeira oportunidade de melhoria foi a divulgação do NSP no Hospital. Com o objetivo de educar e disseminar a cultura de segurança, foi criada uma campanha permanente através da ferramenta LEAN Visual Factory, no sistema de TV interna do hospital, além de fortificação do núcleo com criação de logomarca, além de broches aos membros. Esta implementação teve um custo de duzentos reais; o sistema de TV já estava em uso para outras campanhas de marketing interno (Figura 6).

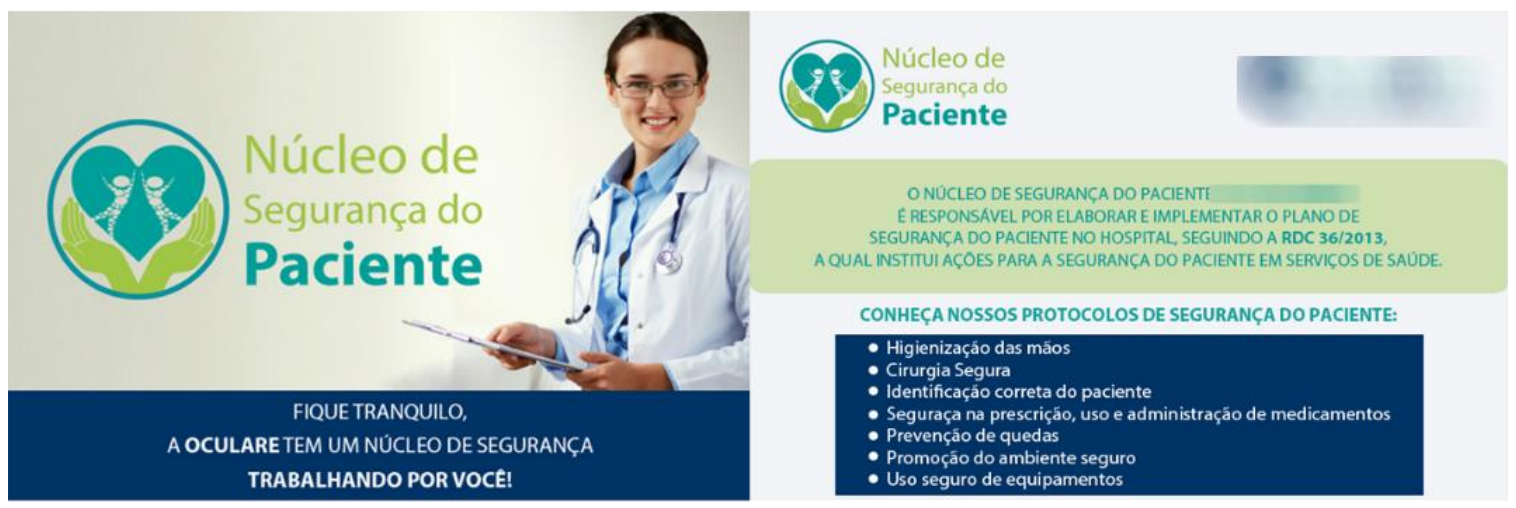

Figura 6. Campanha permanente. 
A notificação de EA, era realizada através de um formulário próprio do NSP, disponível no sistema de gestão da qualidade onde os funcionários tinham que imprimir, preencher e enviar fisicamente ao NSP para análise em reunião, o que gerava grande dificuldade e perda de tempo. A segunda oportunidade de melhoria, foi a utilização das ferramentas Jidoka e Kaizen associadas à TI, para o desenvolvimento de um sistema automatizado que permita o controle instantâneo de notificações, com possibilidade de intervenção imediata por parte dos membros no NSP, e que fosse de fácil acesso. A primeira barreira que enfrentamos para a implantação foi o custo. $O$ desenvolvimento de um software que permitisse que os membros do NSP recebessem as notificações imediatamente, que fosse prático e auto didático para os colaboradores, requeria um investimento inicial cinquenta mil reais, em épocas de crise era inviável. A solução foi criar um site na plataforma WIX (plataforma de design de site gratuito), que permitisse a divulgação do site, educação, informações e notificação. A implantação do site apresentou cinco conteúdos: Página principal (acesso), quem somos (informativo sobre regulamentos e normas legais), objetivo (informativo sobre objetivos do NSP), membros (descrição de cargos e funções dos membros) e notificação (formulário de fácil preenchimento, auto instrutivo, on-line, onde também há informação sobre classificação de EA). Foram gastos 16 horas de trabalho e não houve custos financeiros (Figura 7).
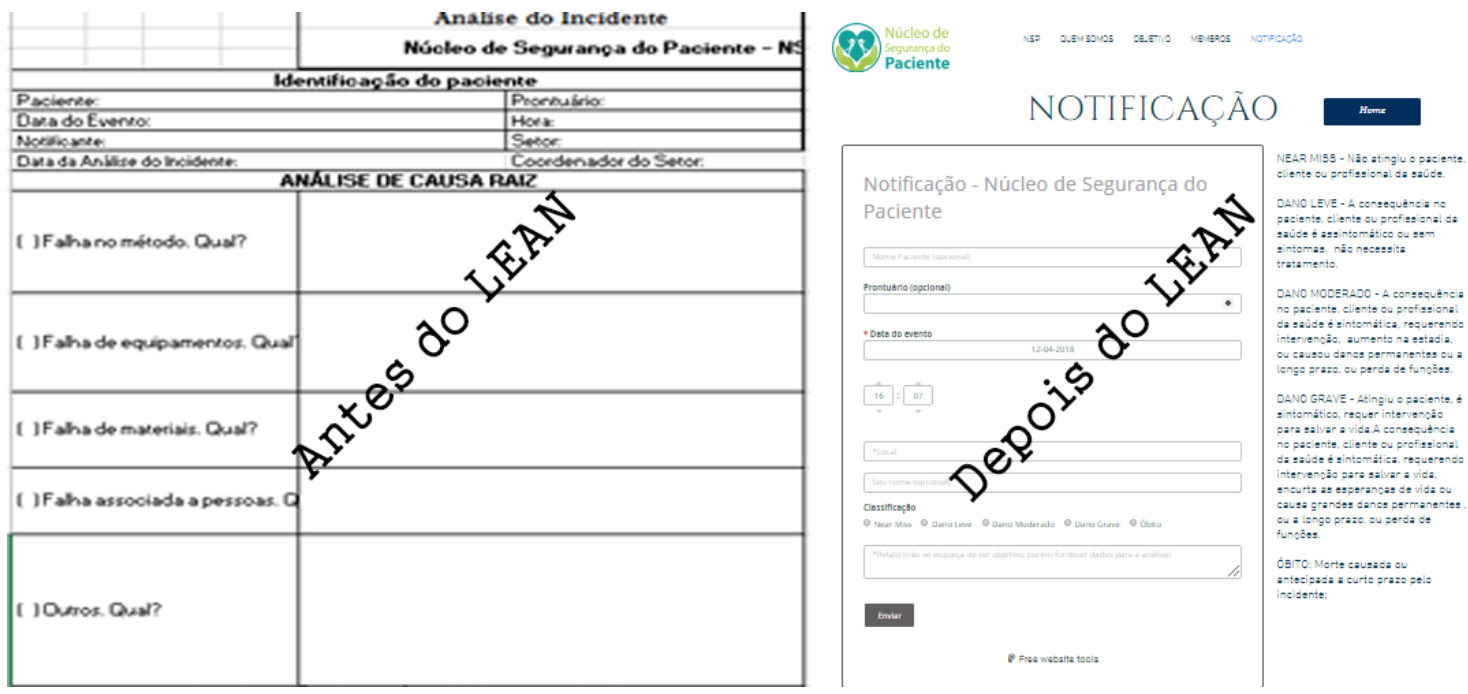

Figura 7. Formulário de notificação NSP - Antes do LEAN e depois do LEAN.

Após implantar duas melhorias, iniciamos a terceira: divulgação, acessibilidade e ensino. Em questões de divulgação e acessibilidade; todos os computadores do hospital passaram a ter em seu desktop o atalho do site do NSP com logomarca, permitindo assim o acesso dos colaboradores ao sistema do NSP com apenas um clique. O site oficial do hospital passou a incorporar um link 
para o site do NSP, permitindo assim, pacientes notificarem também eventos adversos. No aspecto ensino, além da divulgação permanente nas TVs internas, os colaboradores e corpo clínico foram treinados no site onde há todas as informações legais, objetivos, tipos de EA e formulário de notificação de preenchimento auto instrutivo e fácil acesso, diminuindo assim, os problemas levantados pelos colaboradores previamente a implantação da melhoria. Os novos colaboradores e membros do corpo clínico, passaram a ter nos seus treinamentos introdutórios, o treinamento do site e do NSP.

A quarta melhoria foi a notificação instantânea do NSP, que permitiu aos membros notificações em tempo real por e-mail. O formulário, quando preenchido no site, dispara uma mensagem de e-mail para todos os membros com os dados registrados, permitindo com que ações de near miss sejam corrigidas antes que se tornem danos, melhorando o tempo de ação de 27 dias para 1 dia. As informações dos formulários não ficam disponíveis em ambiente web (quando preenchido e enviado aos membros o site elimina a informação sem armazenar nenhum dado), garantindo a segurança das informações.

O resultado destas quatro melhorias, permitiram o maior entendimento e fortificação do pensamento na segurança do paciente, trazendo até o hospital a cultura de segurança com aumento significativo de 357,14\% das notificações de near miss, aumentando a prevenção na cultura de segurança do hospital, tornando o lugar mais seguro para o paciente.

\section{REFERENCIAS}

1) Couto RC, Pedrosa TMG, Roberto BAD, Daibert PB. Anuário da Segurança Assistencial Hospitalar no Brasil. [anuário na internet]. Belo Horizonte: Universidade Federal de Minas Gerais; 2017 [acesso em 02 maio 2019]. Disponível em:

https://www.caism.unicamp.br/PDF/ASAH.pdf

2) World Health Organization (WHO) [homepage na Internet]. Global Priorities for patient safety research. [acesso em 02 de maio de 2019]. Disponível em: http://www.who.int/patientsafety/research/priorities

3) World Health Organization (WHO). Patient safety - a global priority. Bull World Health Organ 2004; 82(12):891-970.

4) Aranaz-Andrés JM, Aibar-Remón C, Limón-Ramírez R, Amarilla A, Restrepo FR, et al. Prevalence of adverse events in the hospitals of five Latin American countries: results of the 'Iberoamerican Study of Adverse Events' (IBEAS). BMJ Qual Saf 2011; 20(12):1043-51.

5) Agência Nacional de Vigilância Sanitária (Brasil). Boletim Informativo. Segurança do Paciente e Qualidade em Serviços de Saúde. Brasília: ANVISA. [Boletim na Internet]. 2011. [acesso em 05 maio 2019]. Disponível em: http://portal.anvisa.gov.br/wps/portal/anvisa/home 
6) Assad ECP. Epidemiologia dos eventos adversos não infecciosos e infecciosos relacionados à assistência em unidade de terapia intensiva de adulto. Belo Horizonte. Tese [Doutorado] - Faculdade de Medicina da Universidade Federal de Minas Gerais; 2011.

7) SINGH H, MEYER AND, THOMAS EJ. The frequency of diagnostic errors in outpatient care: estimations from three large observational studies involving US adult populations. BMJ Qual. Saf 2014; 23(9):72731.

8) THOMAS EJ. et al. Incidence and types of adverse events and negligent care in Utah and Colorado. Med. Care 2000; 38(261): 261-71.

9) Nanji KC et al. Evaluation of perioperative medication errors and adverse drug events. Anesthesiol 2016; 124(1):25-34.

10) Travassos $C$, Porto $S$, Martins $M$, Mendes W. A magnitude financeira dos eventos adversos em hospitais no Brasil. Rev Port Saúde Pública 2010; 10:74-80.

11) Brennan TA et al. Incidence of adverse events and negligence in hospitalized patients. N. Engl. J. Med 1991; 324(6):370-76.

12) ANS - Agência Nacional de Saúde Suplementar (Brasil). Agenda regulatória 2016/2018. [página na internet]. 2018. [acesso em 15 e abr de 2019]. Disponível em: http://www.ans.gov.br/aans/transparenciainstitucional/agenda-regulatoria.

13) ANS - Agência Nacional de Saúde Suplementar. Diretoria de Desenvolvimento Setorial. IN № 61, de 4 de dezembro de 2015. Dispõe sobre a regulamentação dos parágrafos $2^{\circ}$ e $3^{\circ}$ do artigo $7^{\circ}$ da Resolução Normativa - RN no 364, de 11 de dezembro de 2014, que dispõe sobre o Fator de Qualidade a ser aplicado ao índice de reajuste definido pela ANS para prestadores de serviços hospitalares. Rio de Janeiro: ANS, 2015.

14) ANS - Agência Nacional de Saúde Suplementar (Brasil). Portaria №. 529, de $1^{\circ}$ de abril de 2013. Programa nacional de segurança do paciente. Diário Oficial da União 02 abr. 2013; Seção 1.

15) MS - Ministério da Saúde - A Política Nacional de Atenção Hospitalar (PNHOSP) no âmbito do SUS. Portaria de Consolidação no 2, de 28 de julho de 2017. Institui a Consolidação das normas sobre as políticas nacionais de saúde do Sistema Único de Saúde, Capítulo II - Das Políticas de Organização da Atenção à Saúde, Seção I - Das Políticas Gerais de Organização da Atenção à Saúde, Art. 6º - inciso IV, Anexo XXIV (pag. 142 a 145)

16) Brasil. Agência Nacional de Vigilância Sanitária - Anvisa. Resolução da Diretoria Colegiada da Anvisa - RDC no 57, de 16 de dezembro de 2010. Determina o Regulamento Sanitário para Serviços que desenvolvem atividades relacionadas ao ciclo produtivo do sangue humano e 
componentes e procedimentos transfusionais. Diário Oficial da União 17 dez 2010. Alterada por Resolução RDC n 51, de 7 de novembro de 2013. Diário Oficial da União, 13 nov. 2013.

17) Reis CT. A cultura de segurança do paciente: validação de um instrumento de mensuração para o contexto hospitalar brasileiro. Rio de Janeiro. Tese [Doutorado em Saúde Pública]. Fundação Oswaldo Cruz. 2013.

18) Organização Pan-Americana da Saúde - Organização Mundial de Saúde - Opas/OMS; Agência Nacional de Vigilância Sanitária Ministério da Saúde - Anvisa/MS. Manual para Observadores. Brasília. 2008.

19) Aranaz-andrés JM et al. Prevalence of adverse events in the hospitals of five latin american countries: results of the iberoamerican study of adverse events (IBEAS). BMJ Qual. Saf 2011; 20(12):1043-51.

20) Classen D, Resar R, Griffin F et al. Global -trigger toolll shows that adverse events in hospitals may be ten times greater than previously measured. Health Aff 2011; 30:581-9.

21) Braga MA. Influência das infecções relacionadas à assistência no tempo de permanência e na mortalidade hospitalar utilizando a classificação do Diagnosis Related Groups como ajuste de risco clínico. Belo horizonte [Doutorado em Saúde Pública]. Faculdade de Medicina da Universidade Federal de Minas Gerais; 2015.

22) Aspden $P$ et al. Preventing medication errors: Quality chasm series. IOM - Committee on Identifying and Preventing Medication Errors. Washington: The National Academies Press 2007; 544p.

23) Moura MLO, Mendes W. Avaliação de eventos adversos cirúrgicos em hospitais do Rio de Janeiro. Rev. Bras. Epidemiol. 2012; 15(3):523-35.

24) Couto RC. Avaliação do nível de conformidade com requisitos técnicos e legais de segurança assistencial e seus fatores associados em hospitais da rede do Estado de Minas Gerais - 2012. Belo horizonte. Tese [Doutorado em Saúde Pública]. Faculdade de Medicina da Universidade Federal de Minas Gerais; 2014.

25) Daibert PB. Impacto econômico e assistencial das complicações relacionadas à internação hospitalar. Belo horizonte. Tese [Mestrado em Saúde] Faculdade de Medicina da Universidade Federal de Minas Gerais; 2015.

26) Brennan TA et al. Incidence of adverse events and negligence in hospitalized patients. N. Engl. J. Med 1991; 324(6):370-6.

27) Brown $P$ et al. Cost of medical injury in New Zealand: a retrospective cohort study. J. Health Serv. Res. Policy 2002; 7(1):S29-34. 
28) Budnitz DS et al. National surveillance of emergency department visits for outpatient adverse drug events. JAMA 2006; 296(15):1858-66.

29) Reis CT. A cultura de segurança do paciente: Validação de um instrumento de mensuração para o contexto hospitalar brasileiro. Rio de Janeiro. Tese [Mestrado em Saúde] - Fundação Oswaldo Cruz; 2013.

30) Ballé $F$, Ballé M. A Mina de Ouro - Uma transformação lean em romance. São Paulo: Bookman; 2003.

31) Liker JK. The Toyota Way: 14 Management Principles from the World's Greatest Manufacturer. New-York: McGraw Hill; 2004.

32) Womack JP, Jones DT, Roos D. A máquina que mudou o mundo. 14a ed. Rio de Janeiro: Campus; 1992

33) Shingo S. O Sistema Toyota de Produção do ponto de vista da Engenharia de Produção. 2ª Edição. Porto Alegre: Bookman; 1996.

34) Ghinato P. Sistema Toyota de Produção: mais do que simplesmente um Just-inTime. Caxias do Sul: Educs;1996.

35) Buggy JM, Nelson J. Applying Lean Production in Healthcare Facilities. Informedesign 2005; 6:1-5.

36) Chadha R, Singh A, Kalra J. Lean and queuing integration for the transformation of health care processes: A lean health care model. Clinical Governance. An International Journal 2012; 17(3):191- 9.

Recebido: 05 de agosto de 2019. Aceito: 02 de setembro de 2019

Correspondência: Andre Barros Ferreira. E-mail: debarrosnle@hotmail.com

Conflito de Interesses: os autores declararam não haver conflito de interesses.

(C) This is an Open Access article distributed under the terms of the Creative Commons Attribution License, which permits unrestricted use, distribution, and reproduction in any medium, provided the original work is properly cited 